Homology, Homotopy and Applications, vol.21(1), 2019, pp.303-322

\title{
LOCAL FACE RINGS AND DIFFEOMORPHISMS OF QUASITORIC MANIFOLDS
}

\author{
DAVID ALLEN AND JOSÉ LA LUZ
}

(communicated by Donald M. Davis)

\begin{abstract}
In this paper we apply presheaves to develop an invariant that can distinguish diffeomorphism classes of quasitoric manifolds in the category of quasitoric pairs $\mathcal{Q}$. The objects in this category are pointed topological spaces $(M, p)$ where $M$ is a quasitoric manifold and $p$ is a fixed point under the torus action. Maps between pairs are continuous, base-point preserving with respect to a certain topology that depends on the submanifolds of $M$. It is shown that the category of quasitoric manifolds is a subcategory of $\mathcal{Q}$ and then we develop local versions of the Stanley-Reisner ring and the left higher derived functors of the indecomposable functor. We prove that diffeomorphisms between certain objects do not lift to equivalence in the category $\mathcal{Q}$. The main application is geared toward the quasitoric manifolds $\#_{4} \mathbb{C} P^{3}$ with orbit spaces that come from double vertex truncations of the prism that has appeared in the work of Masuda, Panov and their collaborators.
\end{abstract}

\section{Introduction}

In $[\mathbf{B E M P P}]$ it was shown that there is a diffeomorphism of quasitoric manifolds with orbit spaces the double truncated prism. Each such manifold is known to be $\#_{4} \mathbb{C} P^{3}$. However, interestingly, the orbit spaces are not combinatorially equivalent. In fact, of the three, two are combinatorially equivalent and one is not equivalent to the other. This causes a significant obstruction when trying to compare the manifolds using other methods because it is desirable to have a map of quasitoric manifolds that descends to a map of orbit spaces. The implication, of course, is no such map exists, at least not in the category of interest. One way to address this hurdle is to define what it means for manifolds to be diffeomorphic as manifolds with corners. In such a case, it is fairly straightforward to show that the manifolds above are not diffeomorphic in this sense. This definition takes into account the local structure of the polytope and it partly motivated a few of the arguments in this paper. Namely, to formalize the local differences in the orbit spaces in a way that specific calculations can be made.

Received May 10, 2018, revised July 11, 2018, August 21, 2018; published on October 31, 2018. 2010 Mathematics Subject Classification: Primary: 14M25; Secondary: 57N65.

Key words and phrases: quasitoric manifold, toric topology, higher homotopy group, homotopy type, moment angle complex, Davis-Januszkiewicz space, diffeomorphism, rigidity.

Article available at http://dx.doi.org/10.4310/HHA.2019.v21.n1.a14

Copyright (C) 2018, International Press. Permission to copy for private use granted. 
The main contribution of this paper is to show that the manifolds $\#{ }_{4} \mathbb{C} P^{3}$ are not equivalent in the category of quasitoric pairs $-\mathcal{Q}$ (refer to $\S 7$ ). The arguments allow one to compare manifolds with varying orbit spaces without necessarily having a combinatorial map of the orbit spaces. We accomplish this by defining a local version of the Stanley-Reisner ring-a combinatorial invariant that sees the "local" structure of the polytope by analyzing the face rings associated to each vertex in the polytope $P$.

Results proven in $[\mathbf{A}, \mathbf{A L}, \mathbf{A L 2}, \mathbf{A L 3}, \mathbf{A L} 4]$ are used to interpolate between the manifolds and their orbit spaces. Specifically, the left higher derived functors of the indecomposable functor $L_{i} Q(-)$ can be compared between each orbit space by, roughly speaking, lifting the calculations into the cohomology rings of the manifolds through the diffeomorphism and then comparing the local face rings. To make the interpolation compatible, a local version of $L_{i} Q(-)$ that depends on the fixed points of the $T$-action had to be defined for quasitoric manifolds which are part of the datum for a certain generalized toric category.

The comparison depended on carefully counting the number of linearly independent relations among relations that can show up in the orbit spaces. The number of relations was determined by the work of [BP1] through the use of the Koszul resolution and it might be possible to ferret out, using their approach, the rank of the vector space coming from the relations among relations. In $[\mathbf{A L 3}, \mathbf{A L 4}]$ the authors make related calculations directly linking the combinatorics of the orbit space to unstable homotopy theoretic methods. In those works, the ranks of the zeroth, first and second left higher derived functors $\left(L_{i} Q(-)\right.$ for $\left.i=0,1,2\right)$ of the $E_{*}$-face ring (here, $E_{*}$ is a complex orientable theory) were made and applied to $T$-actions. In this paper, a geometric method is developed and applied that allows one to inscribe figures within an $m$-gon that represent these algebraic relations, then we count. The contribution here, is the formalization of the underlying geometry that gives a faithful representation of the algebra.

The paper is organized as follows. In $\S 2$ we re-state certain definitions and the main results. In $\S 3$, key definitions from Toric Topology are listed for the convenience of the reader along with some observations that are used in later parts of the paper. $\S 4$ provides a brief overview of the construction of the left higher derived functors of the indecomposable functor, certain calculations and other items of interest. Some of its key properties that were proven by the authors in earlier work [AL, AL2, AL3, AL4] are listed here too along with the crucial result - Corollary 4.8, whose proof can be found in the appendix. $\S 5$ discusses a new topology on a given quasitoric manifold. $\S 6$ is primarily concerned with the application of presheaves to quasitoric manifolds using the topology defined in $\S 5$. Here, we define "local" versions of the Stanley-Reisner ring and the left higher derived functors. A new category of quasitoric pairs $(M, p)$ ( $M$ is a quasitoric manifold and $p$ is a fixed point under the $T$-action) is defined and used to apply the machinery. The main application used to highlight the tools is given in $\S 7$. We show that the quasitoric manifolds $\#{ }_{4} \mathbb{C} P^{3}$ with the well known orbit spaces coming from the double vertex truncation of the prism $[\mathbf{B E M P P}]$ are not equivalent in the category of quasitoric pairs. 


\section{Acknowledgments}

The first author would like to thank Tony Bahri for providing the images for the polyhedra $P_{i}$ mentioned earlier. He would also like to thank Mikyia Masuda for clarifying vertex cuts and blows-ups in various categories.

\section{Main results}

We recall some key definitions and notation and then list the main results of the paper. Let $P$ be a fixed $n$-dimensional simple convex polytope with vertex set $V(P)$, facet set $\mathcal{F}$ and set of faces $\widehat{\mathcal{F}}$. For $p \in V(P)$ let $\mathcal{F}(p)$ be the collection of facets in $P$ that contain the vertex $p$ and likewise for $\widehat{\mathcal{F}}(p)$. For a commutative ring with unit, $R$, we define a local version of the Stanley-Reisner that depends on a given $p$ as above. The $p$-local face ring is $R(p):=\bigotimes_{F \in \widehat{\mathcal{F}}(p)} R(F)$ where $R(F)$ is the $R$-face ring of $F$ (see definition 3.10 for the definition of $R(F)$ ).

For a given quasitoric manifold $\pi: M \rightarrow P$ and $F$ a face of $P$, a topology on $M$ is constructed whose basis depends on the submanifolds $M_{F}$ where $M_{F}=\pi^{-1}(F)$. This is then used to define a category $\mathcal{Q}$ of quasitoric pairs which contains the category of quasitoric manifolds as a sub-category. A quasitoric pair consists of a pair $(M, p)$ where $M$ is a quasitoric manifold and $p$ belongs to the set of fixed points of $M$ under the $T$-action; namely, $p \in \pi^{-1}(V(P))$. Maps in the category $\mathcal{Q}$ are maps of $M$ which are continuous with respect to the topology mentioned in $\S 5$ and send the fixed point $p$ to another fixed point. Within this generalized category, we only partially consider the torus action. Namely, it is encoded in the basic sets in the topology and the fixed points. Given the topology mentioned above, we define fixed-point dependent left higher derived functors by considering a presheaf $\mathfrak{F}$. For additional details refer to $\S 6$. Roughly speaking, the presheaf $\mathfrak{F}$ maps each basic open set, a sub-quasitoric manifold $M_{F}$ for a given face $F$ of $P$, to the cohomology ring $H^{*}\left(M_{F}\right)$.

A local version of the left higher derived functors of a quasitoric pair are defined in $\mathcal{Q}$, what we deem fixed point higher derived functors of the indecomposable functor. For $j \geqslant 0$,

$$
L_{j} Q(M, p):=L_{j+1} Q\left(\bigotimes_{U \in \mathcal{B}_{p}} \mathfrak{F}(U)\right) \cong \bigoplus_{U \in \mathcal{B}_{p}} L_{j+1} Q(\mathfrak{F}(U)),
$$

where $\mathcal{B}_{p}$ is defined in Notation 6.6. There is an isomorphism of these fixed-point local constructions that re-frame constructions/theorems that appeared in earlier work of the authors $[\mathbf{A L}, \mathbf{A L 2}, \mathbf{A L 3}, \mathbf{A L 4}]$.

Theorem 6.10. Let $(M, p)$ be a quasitoric pair. Then for $j \geqslant 0$,

$$
L_{j} Q(M, p) \cong L_{j+1} Q(R(p)) \cong \bigoplus_{F \in \widehat{\mathcal{F}}(p)} L_{j+1} Q(R(F) 0) .
$$

The methods above allow one to move between certain spaces and their orbit spaces even though there may not be a combinatorial map between the orbit spaces. More specifically, one may lack a map of quasitoric manifolds in the category of quasitoric manifolds but there is a map in the category of quasitoric pairs. In earlier work $[\mathbf{A}, \mathbf{A L}, \mathbf{A L 3}, \mathbf{A L} 4]$, we proved there is an isomorphism of the left higher derived 
functors of the indecomposable functor of $H^{*}\left(M_{P}\right)$ and $H^{*}\left(B_{T} P\right)$ for a given simple convex polytope $P$. In the case where there is an isomorphism $H^{*}\left(M_{P}\right) \cong H^{*}\left(M_{Q}\right)$ for two given quasitoric manifolds $M_{P}$ and $M_{Q}$ with orbit spaces $P$ and $Q$, these results allow one to frame rigidity questions in another context. Here, we have a method to descend from the global object to specific structures within the orbit space and the manifold. More is true, although not pursued in the current paper (currently a work in progress) and that is, certain rigidity problems of the orbit spaces can now be analyzed using these methods and done so in a way that depends on fixed points.

These methods are applied to the quasitoric manifolds $\#_{4} \mathbb{C} P^{3}$ with orbits spaces being obtained from a double vertex truncation of the prism. All of these manifolds are diffeomorphic $[\mathbf{B E M P P}]$ and not all of the orbits spaces have combinatorial maps between them in such a way that is consistent with having a map of quasitoric manifolds. One can declare that the manifolds are not diffeomorphic as manifolds with corners, which of course, takes local information into account.

Before stating the main result, we recall some notation. For $i=1,2$, let $\pi_{i}: M_{i} \rightarrow$ $P_{i}$ be quasitoric manifolds with orbit spaces $P_{1}$ and $P_{2}$ resp. Using notation that is well accepted, $P_{1}=6^{2} 4^{3} 3^{2}$ and $P_{2}=6^{1} 5^{2} 4^{2} 3^{2}$. The polytope $P_{1}$ has as facets, two 6 -gons, three 4-gons and two 3 -gons and $P_{2}=6^{1} 5^{2} 4^{2} 3^{2}$ has one 6 -gon, two 5 -gons, two 4 -gons and two 3 -gons as facets. Each manifold, $M_{1}$ and $M_{2}$ is diffeomorphic to $\#_{4} \mathbb{C} P^{3}$. Additional details can be found in $[\mathbf{B E M P P}]$. From $\S 6$, we recall the definition of equivalence in the category $\mathcal{Q}$. Namely, two quasitoric pairs $(M, p)$ and $(N, q)$ are equivalent in the category $\mathcal{Q}$ if there is a base-point preserving homeomorphism in the topology defined by $\mathcal{Q}$. The main result is:

Theorem 7.2. The quasitoric pairs $\left(M_{1}, p_{1}\right)$ and $\left(M_{2}, p_{2}\right)$ are not equivalent in $\mathcal{Q}$ for any $p_{1} \in \pi_{1}^{-1}\left(V\left(P_{1}\right)\right)$ and $p_{2} \in \pi_{2}^{-1}\left(V\left(P_{2}\right)\right)$.

The appendix contains a counting argument that is used in proving the main result. This requires enumerating the relations and relations among relations in the StanleyReisner ring of the $m$-gon. We note that [BP1] uses the Koszul resolution to count, at least the relations, in a very concrete way, but algebraically. In that paper, the count is given by a certain bi-graded module $R^{-1,2 j}$ where $j$ depends on the missing face. Our contribution, in this context, is to develop a geometric approach based on inscribing figures into the $m$-gon that encode linear dependence (and independence) then to count these figures in an orderly way. Although not explicitly written down, our calculations agree with $[\mathbf{B P 1}]$ in the cases under consideration and our approach gives a faithful geometric interpretation that can be used to possibly determine higher order relations among relations (see Appendix for a precise definition).

\section{Toric topology}

In this section we will highlight some well-known constructions in Toric Topology. Some excellent references for this material would include the conference proceedings [BR2] and the AMS book [BP1]. Much this section can be found in the paper [AL3] and it is included here for the convenience of the reader.

For the applications needed in this paper, it is assumed that $P$ is a simple convex polytope with $m$ facets $F_{1}, \ldots, F_{m}$ which as a set, we label as $\mathcal{F}$. The polytope $P$ 
is $q \geqslant 1$ neighborly if the intersection of any $q$ facets is not empty [BP1, p. 11] and we further assume for the remainder of this paper that $P$ is $q \geqslant 1$ neighborly. The collection of faces of $P$ will be denoted by $\widehat{\mathcal{F}}$. We will use the notation $V(P)$ to denote the set of vertices of $P$. For $p \in V(P), \widehat{\mathcal{F}}(p)$ is the set of faces containing $p$ and $\mathcal{F}(p)$ is the set of facets containing $p$. The torus $T^{n}$ refers to the $n$-dimensional topological torus and $B T^{n}$ its classifying space. When there is no chance of confusion we simply write $T$ instead of $T^{n}$.

Using the perspective of derived forms the notion of a quasitoric manifold and related spaces can be described. In fact, derived forms are formulated in a much more general setting, where sets more general than polyhedra are used [BR2]. Following Buchstaber and Ray's exposition, there is a map

$$
P \stackrel{\lambda}{\longrightarrow} T\left(T^{n}\right)
$$

where $T\left(T^{n}\right)$ is the lattice of subtorii of the torus ordered by inclusion and the topological structure is induced from the lower limit topology. $\lambda$ sends $q \in P$ to a certain subtorus, $\lambda(q)$. The derived space is by definition the following quotient space

$$
D(\lambda)=\left(T^{n} \times P\right) / \sim,
$$

where $(g, q) \sim(h, q)$ if and only if $g^{-1} h \in \lambda(q)$. It can be shown that $\sim$ is an equivalence relation. The elements in $D(\lambda)$ are equivalence classes $[g, q]$ for which there is a canonical action of the torus on $D(\lambda)$ via multiplication on the first coordinate. The orbit space of this action is $P$; using the atlas $\left\{U_{v}\right\}$ given by $[\mathbf{B P} 1$, p. 63 , construction 5.8], it can be shown that the torus action is locally standard [DJ, p. 420] (here locally standard is referred to as locally isomorphic to the standard representation).

Remark 3.1. Davis and Januszkiewicz [DJ] refer to the subgroup $G_{F}$ of the lattice $\mathbb{Z}^{m}$ "determined by $T^{n}$ and $\lambda$ " cf., p. 423, 1.5. In addition, $\lambda$ determines a map between integer lattices $\mathbb{Z}^{m} \rightarrow \mathbb{Z}^{n}$. Note that this map is also referred to as "lambda" in many research articles. Recall, to each facet $F_{j}$ of $P$, one associates a certain circle subgroup, say $T_{\lambda}\left(F_{j}\right)$. In [BP1] these subtorii were made explicit, see p. $64,(5.3)$. Each such torus subgroup gives rise to a facet vector $\lambda_{i j} \in \mathbb{Z}^{n}$, for $1 \leqslant i \leqslant n, 1 \leqslant j \leqslant$ $m$. These vectors are indexed by the facets and from this and the discussion above, a function can be defined from the set of facets of $P, \mathcal{F}$, to the integer lattice whose dimension depends on the dimension of $P$. This is how the characteristic function $\mathcal{F} \rightarrow \mathbb{Z}^{n}$ is obtained and by a simple identification, the function $\mathbb{Z}^{m} \rightarrow \mathbb{Z}^{n}$ can be derived.

Briefly summarizing condition $(*)$ in $[\mathbf{D J}]$, we have the requirement that each codimension $k$-face of $P$ gives a $k$-dimensional direct summand of $\mathbb{Z}^{n}$.

To obtain a quasitoric manifold (or Toric manifold in the language of $[\mathbf{D J}]$ ), one must impose conditions on $\lambda$ and a smooth structure on $D(\lambda)$ cf. $[\mathbf{D J}]$. First, $\lambda$ associates a circle to the interior of a facet. Hence, if $F_{j}$ is a facet, then $T_{\lambda}\left(F_{j}\right)$ is a circle. For additional details, see [BP1, p. 64, (5.3)]. Second, if $F$ is a codimension $k$-face, that is, the intersection of $k$ facets, then the torus subgroup associated to the interior of $F$ is the product of those coordinate tori coming from each of the facets whose intersection is the face $F$. Following the exposition in $[\mathbf{B P 1}]$ and using their notational conventions suppose $G$ is a codimension $d$ face $\left(G=\bigcap_{k=1}^{s} F_{j_{k}}\right)$, then the 
following map is an isomorphism onto the image $T_{\lambda}(G)$ :

$$
i m\left(\prod_{1 \leqslant k \leqslant d} T_{\lambda}\left(F_{j_{k}}\right)\right) \longrightarrow T^{n} .
$$

Finally, it is required that the $\operatorname{Ker}$ of $\lambda$ partitions $P$ by the interior of the faces. By a Quasitoric manifold $M^{2 n}(\lambda)$ one means the derived space $D(\lambda)$ where $\lambda$ is subject to the conditions described above.

Notation 3.2. Throughout the paper the dimension of a quasitoric manifold may be dropped as well as any reference to $\lambda$ when the context is clear. For example, we may write $M$ or $M(\lambda)$ instead of $M^{2 n}(\lambda)$. In addition, if $M$ (or $M(\lambda)$ ) is a quasitoric manifold with orbit space $P$, then it is common to say that $M$ is a quasitoric manifold over $P$ or $M$ sits over $P$ (this parlance is also adopted in [BR2]) and we shall simple write $\pi: M \rightarrow P$ (here it is implied that $P$ is the orbit space).

Examples of quasitoric manifolds would include the following: $\mathbb{C} P^{n}$ with orbit space $\Delta^{n}$. Buchstaber and Ray $[\mathbf{B R}]$ show that the $2 n$-dimensional manifold $-B_{n}$ of all bounded flags in $\mathbb{C}^{n+1}$ is a quasitoric manifold over $I^{n}$. Buchstaber and Ray $[\mathbf{B R}]$ show that $C P^{n} \sharp C P^{n}$ is a quasitoric manifold over $\Delta^{1} \times \Delta^{n-1}$ by defining a connect sum operation on the level of the polytopes. Orlik and Raymond $[\mathbf{O R}]$ classified four dimensional quasitoric manifolds that sit over polygons and showed that they are connect sum of the Hirzebruch surface with connect sums of $\mathbb{C} P^{2}$.

Given $\pi: M \rightarrow P$, there is an action $T \times M \rightarrow M$ such that the orbit space is $P$. The fixed points of this action are zero dimensional manifolds corresponding to the pre-images of the vertices of $P$. Namely, for $p \in V(P), \pi^{-1}(p)$ is a fixed point under the $T$-action.

We now introduce the notion of a certain generalized map of quasitoric manifolds. For $m \in M$ and $t \in T$ the $T$-action on $M$ will be denoted by $t * m$. We take as our motivation Definition 5.3 from [BP1].

Let $P_{1}$ and $P_{2}$ be two polyhedra. We say $f: P_{1} \rightarrow P_{2}$ is a pseudo-combinatorial map if $f$ maps vertex to vertex and for each face $G \subseteq P_{2}, f^{-1}(G)$ is a union of faces in $P_{1}$.

Remark 3.3. If $f: P_{1} \rightarrow P_{2}$ is a combinatorial map, then it is a pseudo-combinatorial map.

Definition 3.4. For $1 \leqslant i \leqslant 2$, let $T^{n_{i}}$ be the $n_{i}$-torus that acts on the quasitoric manifold $M_{i}$ with orbit space $P_{i}$. A generalized equivariant map of quasitoric manifolds is a pair of maps $(\phi, \psi)$ where $\phi: M_{1} \rightarrow M_{2}$ and $\psi: T^{n_{1}} \rightarrow T^{n_{2}}$ such that the following diagram commutes:

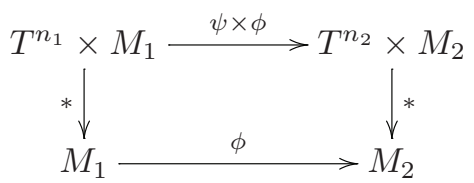

where the vertical maps are the actions of the corresponding tori and the map $\phi$ induces a pseudo-combinatorial map $\phi_{*}: P_{1} \rightarrow P_{2}$.

Notation 3.5. If there is no possibility confusion, then we say that the generalized equivariant map $(\phi, \psi)$ is a map from $M_{1}$ to $M_{2}$. 
Remark 3.6. In Definition 3.4 we do not assume that such a map induces a map of the underlying polyhedra. Namely, the induced map $\phi_{*}$ does not necessarily preserve the dimension of a face.

Proposition 3.7. Suppose, for $i=1,2, M_{i}$ is a quasitoric manifold with orbit space $P_{i}$ and $(\phi, \psi)$ is a generalized equivariant map from $M_{1}$ to $M_{2}$. Then the map $\phi$ is well defined.

Proof. Let $m_{1} \in M_{1}$ and write its orbit as $\left[m_{1}\right]$. Define $\phi\left(\left[m_{1}\right]\right)=\left[\phi\left(m_{1}\right)\right]$. We only need to prove that $\phi$ is well defined. Suppose $x \in\left[m_{1}\right]$, then there exists $t^{\prime} \in T^{n_{1}}, m^{\prime} \in$ $M_{1}$ such that $x=t^{\prime} * m^{\prime}$. By the commutativity of the diagram we have $\phi(x)=$ $\phi\left(t^{\prime} * m^{\prime}\right)=\psi\left(t^{\prime}\right) * \phi\left(m^{\prime}\right) \in\left[\phi\left(m_{1}\right)\right]$. Hence, $\phi$ is well defined.

An important case arises when $n_{1}=n_{2}, P_{1}=P_{2}$ with $\phi: M_{1} \rightarrow M_{2}$ is a homeomorphism such that induced map on the orbit spaces is the identity, then the quasitoric manifolds are equivalent [DJ]. In [BP1, p. 65, Definition 5.13], the notion of $\psi$ equivariant diffeomorphism of quasitoric manifolds with the same orbit space is described. In both of these formulations $\psi \in \operatorname{Aut}(q T)$. In this case there is a commutative diagram where $T=T^{n_{i}}$.

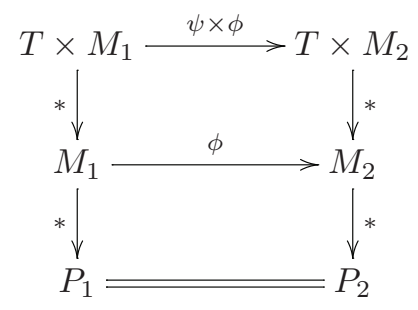

Definition 3.8. The category $\mathcal{G}$ is the category where the objects are quasitoric manifolds and maps are generalized equivariant quasitoric maps.

Maps in the category $\mathcal{G}$ are those described in Definition 3.4 and they are equivariant maps that send a fixed point to a fixed point without necessarily descending to a map of polyhedra. The maps in the category $\mathcal{G}$ are weaker than the maps in the category of quasitoric manifolds.

Given a set $X$ endowed with an action of a group $G$, the Borel Construction can be used to replace the orbit space $X / G$, by a space $E G \times_{G} X$ which is homotopy equivalent to the orbit if the action is free, the latter fitting into a fibration. In the case of a quasitoric manifold $M$, we have the following:

Definition 3.9. Let $M$ be a quasitoric manifold. The Borel space $B_{T} M$ is the identification space

$$
E T \times M / \sim=E T \times_{T} M,
$$

where the equivalence relation is defined by: $(e, x) \sim\left(e g, g^{-1} x\right)$ for any $e \in E T$ and $x \in M, g \in T$.

Notational convention: Sometimes the notation $B_{T} P$ appears in the literature instead of $B_{T} M$. The orbit space of the $T$-action on a quasitoric manifold $M$ can be identified with $P$, this should give some insight into the notational convention. When 
the context is clear, the notation $B_{T} P$ will be used instead of $B_{T} M$. The following fibration is well known $[\mathbf{B P 1}, \mathbf{D J}]$ :

$$
M(\lambda) \longrightarrow B_{T} P \longrightarrow B T .
$$

The face ring is an invariant that will be useful in the sections that follow. Recall,

Definition 3.10. Let $F_{1}, \ldots, F_{m}$ be the facets of $P$. For a fixed commutative ring $R$ with unit we have

$$
R(P)=R\left[v_{1}, \ldots, v_{m}\right] /\left(v_{i_{1}} \cdots v_{i_{k}} \mid F_{i_{1}} \cap \cdots \cap F_{i_{k}}=\emptyset\right),
$$

where $\left|v_{i}\right|=2$ are indexed by the facets and the ideal $I$ is generated by square free monomials coming from trivial intersection of facets.

For example, if $P=\partial \Delta^{2}$, then $\mathbb{Z}(P)=\mathbb{Z}\left[v_{1}, v_{2}, v_{3}\right] /\left(v_{1} v_{2} v_{3}\right)$. Sometimes we refer to $R(P)$ as the Stanley-Reisner algebra and $I$ the Stanley-Reisner ideal.

Theorem 3.11. Let $P$ be a simple convex polytope, then $H^{*}\left(B_{T} P\right) \cong \mathbb{Z}(P)$.

The proof can be found in $[\mathbf{D J}]$. An easy application of the Atiyah-Hirzebruch spectral sequence gives the following generalization of Theorem 3.11. Namely, for $P$ as above, $H^{*}\left(B_{T} P ; R\right) \cong R(P)$. Additional details can be found in $[\mathbf{A}]$.

Before we proceed we give a result that will be used in $\S 4$.

Lemma 3.12. Let $P$ be a fixed simple convex polytope. For $i=1,2$, let $M_{F_{i}}$ be quasitoric manifolds over the faces $F_{i}$ of $P$. Further assume $M_{F_{1}} \cap M_{F_{2}} \neq \emptyset$, then $M_{F_{1}} \cap M_{F_{2}}$ is a quasitoric manifold over the smallest face of $P$ contained within $F_{1}$ and $F_{2}$.

Proof. Recall, each quasitoric manifold $M_{i}=\left(T^{k_{i}} \times F_{i}\right) / \sim$ is a union of sets of the form $\left(T^{k_{i}} \times U_{v}\right) / \sim$, the latter homeomorphic to a copy of $\mathbb{C}^{r}$ for some $r$. The sets $U_{v}$ are those described in $[\mathbf{B P} \mathbf{1}]$. On the intersection form the union of those sets to give $U=\left\{U_{v^{\prime}}\right\}$ which is an atlas that comes from the smallest face contained within both faces. Form the quotient $\left(T^{r^{\prime}} \times U\right) / \sim$ where $r^{\prime}$ is the dimension of the smallest face. The corresponding quasitoric manifold is $M_{F_{1}} \cap M_{F_{2}}$.

\section{Higher derived functors}

We assume that $R$ is a commutative ring with unit and that all (graded) $R$-algebras are connected with unit $\eta: R \rightarrow A$. It is further assumed that all algebras are free $R$-modules. Let $\bar{A}=\operatorname{Coker}(R \stackrel{\eta}{\rightarrow} A)$. The rank of an $R$-module $M$ will be denoted by $r k(M)$. Recall that the $R$-face ring of $P$ is written as: $R(P)$. For the convenience of the reader we list here material from $[\mathbf{A L}, \mathbf{A L 2}$, AL3, AL4] on certain left higher derived functors that are used in the sequel. Additional information can be found in those papers.

Definition 4.1. Let $A$ be an $R$-algebra. The module of indecomposables of $A$ is defined and denoted by

$$
Q(A)=\bar{A} / \bar{A}^{2}
$$


$Q$ defines a non-additive functor from the category of (graded) $R$-algebras to the category of (graded) $R$-modules.

Let $F$ be the free, commutative algebra functor with unit over $R$. It is a functor from the category of free $R$-modules to the category of $R$-algebras and comes equipped with a natural transformation $s: 1 \rightarrow F$. There is a diagram that describes the universal property of $F$. If $M$ is a free $R$-module and $A$ is an $R$-algebra with an $R$-module map $f: M \rightarrow A$, then there is a unique $R$-algebra map $\bar{f}: F(M) \rightarrow A$ such that the following diagram commutes:

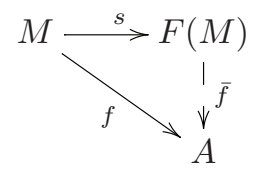

If $M=J(A)$ where $J$ is the forgetful functor from the category of $R$-algebras to the category of $R$-modules then we have $f=i d, s_{-1}=s$ and $d_{0}=\overline{i d}$, then we obtain an augmented simplicial object over the category of $R$-algebras (to simplify notation we will omit the functor $J$ from now on): $\mathbf{F}^{\bullet}(A)$

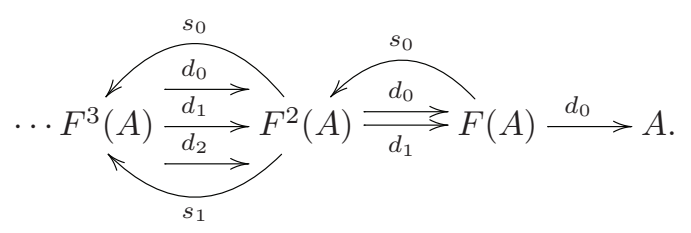

The $d_{i}=F^{n}\left(\left(d_{0}\right)_{F^{n-i}(A)}\right): F^{n}(A) \rightarrow F^{n-1}(A)$ for $0 \leqslant i \leqslant n$ and for $0 \leqslant i \leqslant n$ we have $s_{i}=F^{n}\left(\left(s_{-1}\right)_{F^{n-i}(A)}\right): F^{n}(A) \rightarrow F^{n}(A)$. Applying the functor $Q$ we obtain an un-augmented chain complex, $\operatorname{ch}_{u}\left(Q \mathbf{F}^{\bullet}(A)\right)$ with $\delta_{n}=\sum_{i=0}^{n}(-1)^{i} Q\left(d_{i}\right)$.

\section{Definition 4.2.}

$$
L_{i} Q(A ; R)=H_{i}\left(\operatorname{ch}_{u}\left(Q \mathbf{F}^{\bullet}(A)\right)\right) .
$$

If the ring $R$ is fixed, then we simply write $L_{i} Q(A)$.

Notation 4.3. From this point forward, when the context is clear, we abbreviate $L_{i} Q(-; R)$ by $L_{i} Q(-)$.

The basic properties of these functors are summarized below:

Theorem 4.4. For any $R$-algebras $A, B$ and $C$,

i) $L_{0} Q(A)=Q A$

ii) If $A$ is a free algebra, then $L_{i} Q(A)=0$ for $i>0$

iii) $L_{i} Q\left(A \otimes_{R} B\right) \cong L_{i} Q(A) \oplus L_{i} Q(B)$

The interested reader can refer to $\S 3$ of $[\mathbf{A L 3}]$ for proofs and calculations.

Definition 4.5. Let $A=R\left[v_{1}, \ldots, v_{m}\right] /\left(r_{1}, \ldots, r_{l}\right)$ where $r_{i}$ are square-free monomials. If $r_{i}=v_{i_{1}} \cdots v_{i_{k}}$ then let $I\left(r_{i}\right)=\left\{i_{1}, \ldots, i_{k}\right\}$. When reference is made to the intersection of $r_{i}$ and $r_{j}$ we mean $I\left(r_{i}\right) \cap I\left(r_{j}\right)$. Define $I=I\left(r_{i}\right) \cup I\left(r_{j}\right)$ along with the products $\widehat{x_{i}}=\prod_{s \in\left(I \backslash I_{i}\right)} v_{s}$ and $\widehat{x_{j}}=\prod_{s^{\prime} \in\left(I \backslash I_{j}\right)} v_{s^{\prime}}$. We observe, for $i \neq j, \widehat{x}_{i}$ and $\widehat{x}_{j}$ are square-free monomials in $A$. With this notation, a relation among relations in $A$ is a polynomial of the form: $\rho_{i j}=r_{i} \widehat{x}_{i}-r_{j} \widehat{x}_{j}=0$. 
Theorem 4.6. Let $A=R\left[v_{1}, \ldots, v_{m}\right] /\left(r_{1}, \ldots, r_{n}\right)$ then:

i) $L_{0} Q(A)=\operatorname{span}_{R}\left\{v_{1}, \ldots, v_{m}\right\}$

ii) $L_{1} Q(A)=\operatorname{span}_{R}\left\{r_{1}, \ldots, r_{m}\right\}$

iii) $L_{2} Q(A)=\operatorname{span}_{R}\left\{\rho_{i j}\right\}$ where $\rho_{i j}$ are relations among relations of minimal degree with non-empty intersection.

Proof. See $[$ AL3 $]$ for details.

Remark 4.7. Referring to item (iii) in Theorem 4.6 the relations among relations are built upon minimal relations. By this, it is intended to mean those relations that are not divisible by any relation of smaller degree.

Let $P$ be an $m$-gon, then the dual one-dimensional simplicial complex shall be written $K_{m}$.

Corollary 4.8. For $m \leqslant 6$, the rank of $L_{2}\left(Q\left(R\left(K_{m}\right)\right)\right)$ is listed in the following table:

\begin{tabular}{|c|c|c|c|c|}
\hline$m$ & 3 & 4 & 5 & 6 \\
\hline$r k\left(L_{2} Q\left(R\left(K_{m}\right)\right)\right)$ & 0 & 0 & 5 & 16 \\
\hline
\end{tabular}

Proof. The result follows from Theorem A.2 in the appendix.

The next result was proven in $[\mathbf{A L 3}, \mathbf{A L 4}]$ and it concerns the relations between the isomorphism of the higher derived functors described by Definition 4.2 and certain coefficient rings.

Theorem 4.9. For any complex orientable theory $E$ with coefficients $E_{*}$ we have

$$
L_{i} Q E^{*}\left(M(\lambda) ; E_{*}\right) \cong L_{i} Q E^{*}\left(B_{T} P ; E_{*}\right)
$$

for $i \geqslant 1$.

\section{A new topology for $M$}

Let $P$ be an $n$-dimensional simple convex polytope with vertex set $V(P)$ and set of faces $\widehat{\mathcal{F}}$. If $\pi: M \rightarrow P$ is a quasitoric manifold and $F \in \widehat{\mathcal{F}}$, then $M_{F}$ is a sub-quasitoric manifold of $M[\mathbf{D J}]$. We will now prove that the set $\mathcal{B}=\left\{M_{F} \mid F \in \widehat{\mathcal{F}}\right\}$ forms a basis for a topology on $M$ which, in the sequel, will be written $\mathcal{T}_{M}$.

Lemma 5.1. $\mathcal{B}$ forms a basis for a topology on $M$.

Proof. It is clear that $M$ is the union of sets in $\mathcal{B}$. Consider two quasitoric manifolds $M_{F_{i}}$ and $M_{F_{j}}$ corresponding to two faces, $F_{i}$ and $F_{j}$ of $P$. If their intersection is empty, then the basis condition is satisfied trivially. Suppose the intersection is not trivial and assume further that $x \in M_{F_{i}} \cap M_{F_{j}}$. By Lemma 3.12 the face coming from the intersection is the smallest that contains the point $x$ that is a sub-face of both $F_{i}$ and $F_{j}$. The corresponding quasitoric manifold is a submanifold of $M_{F_{i}}$ and $M_{F_{j}}$, hence is in the intersection. This completes the proof. 
Remark 5.2. We note that for a given quasitoric manifold $M$ there are two topologies. The first comes from the definition and the second is the one whose open sets belong to $\mathcal{T}_{M}$. In the latter case, the basic open sets are the manifolds themselves. We do not assert any compatibility of the topologies at this stage. Furthermore, the open sets in the topology coming from elements of $\mathcal{B}$ do not necessarily preserve the quasitoric structure. Namely, we are not implying the action behaves well, nor are we implying any map is a map of quasitoric manifolds.

\section{Presheaves}

Recall, $R$ is a commutative ring with unit and the basis elements in $\mathcal{B}$ are quasitoric sub-manifolds $M_{F}$ that arise as the pre-images of the faces $F \subseteq P$ under the map $\pi: M \rightarrow P$.

For a given fixed quasitoric manifold $M$, the topology $\mathcal{T}_{M}$ gives rise to a category in the usual way. The objects are the open sets and the maps are the continuous inclusions. Rather than introduce additional notation, we simply refer to this category as $\mathcal{T}_{M}$.

Definition 6.1. We define a functor $\mathfrak{F}$ from $\mathcal{T}_{M}$ to the category of $R$-algebras defined by

$$
\mathfrak{F}(U ; R)=\mathfrak{F}(U)= \begin{cases}R & U \notin \mathcal{B} \text { or } U=M, \\ H^{*}(U ; R) & U \in \mathcal{B} .\end{cases}
$$

It is readily verified that $\mathfrak{F}$ is a functor.

Note 6.2. A continuous map $f: M_{1} \rightarrow M_{2}$ where $M_{i}$ is given the topologies $\mathcal{T}_{M_{i}}$ induces a map $f^{-1}: \mathcal{T}_{M_{2}} \rightarrow \mathcal{T}_{M_{1}}$. Since cohomology is contravariant, this in turn, will give an induced map $f_{*}^{-1}: \mathfrak{F}_{1} \rightarrow \mathfrak{F}_{2}$ where $\mathfrak{F}_{i}$ is the presheaf over $\mathcal{T}_{M_{i}}$ for $i=1,2$.

Recall, for $p \in V(P), \widehat{\mathcal{F}}(p)$ is the collection of facets in $P$ whose intersection is $p$. We can now define a local version of face ring that depends on the point. For $p \in V(P)$, the $p$-local face ring (or p-local Stanley-Reisner ring) is:

$$
R(p)=\bigotimes_{F \in \widehat{\mathcal{F}}(p)} R(F)
$$

Definition 6.3. Given a quasitoric manifold $M$ over $P$ and a base point $p \in \pi^{-1}(V(P))$, we call $(M, p)$ a quasitoric pair. Let $\mathcal{Q}$ be the category whose objects are quasitoric pairs and the maps are continuous, base-point preserving with respect to $\mathcal{T}_{M}$.

Note 6.4. There is a clear relationship between the categories $\mathcal{G}$ and $\mathcal{Q}$ (see $\S 3$ ). Suppose $\pi_{i}: M_{i} \rightarrow P_{i}$ are quasitoric manifolds for $i=1,2$. Then by Proposition 3.7 we have a map $\phi_{*}: P_{1} \rightarrow P_{2}$. This implies $\phi:\left(M_{1}, \mathcal{T}_{M_{1}}\right) \rightarrow\left(M_{2}, \mathcal{T}_{M_{2}}\right)$ is continuous and $\phi\left(\pi_{1}^{-1}\left(V\left(P_{1}\right)\right)\right) \subseteq \pi_{2}^{-1}\left(V\left(P_{2}\right)\right)$. Then for any $p \in \pi_{1}^{-1}\left(V\left(P_{1}\right)\right)$ the map $\phi:\left(M_{1}, p\right) \rightarrow$ $\left(M_{2}, \phi(p)\right)$ is a map in $\mathcal{Q}$.

Two quasitoric pairs $(M, p)$ and $(N, q)$ are equivalent in the category $\mathcal{Q}$ if there is a base-point preserving homeomorphism in the topology defined by $\mathcal{Q}$. 
Remark 6.5. Equivalence in $\mathcal{G}$ implies equivalence in $\mathcal{Q}$ and we note that in the category $\mathcal{Q}$, the preimage of a basic set $M_{F}$, where $F$ is a face, could be the union of submanifolds. On the level of polyhedra this would imply the image of a union of faces is a face. This is not a map of polytopes. Therefore, an equivalence in $\mathcal{Q}$ does not imply the manifolds are diffeomorphic as manifolds with corners.

Notation 6.6. For $p \in \pi^{-1}(V(P))$ we let

$$
\mathcal{B}_{p}=\{U \in \mathcal{B} \mid p \in U\} .
$$

Definition 6.7. Let $(M, p)$ be an object in the category $\mathcal{Q}$. Then for $j \geqslant 0$, the fixed point higher derived functors of the indecomposable functor of $(M, p)$ are defined as follows:

$$
L_{j} Q(M, p)=L_{j+1} Q\left(\bigotimes_{U \in \mathcal{B}_{p}} \mathfrak{F}(U)\right) \cong \bigoplus_{U \in \mathcal{B}_{p}} L_{j+1} Q(\mathfrak{F}(U))
$$

Remark 6.8. Observe that the shift in dimension ensures that we have an isomorphism between the left higher derived functors of the indecomposable functor of the Stanley-Reisner ring and the quasitoric manifold as described in Theorem 4.9.

Proposition 6.9. For any object $(M, p)$ in $\mathcal{Q}$ and $j \geqslant 0, L_{j} Q(-)$ is a covariant functor from $\mathcal{Q}$ to the category of graded $R$-modules.

Proof. Let $\left(M_{1}, p_{1}\right)$ and $\left(M_{2}, p_{2}\right)$ be quasitoric pairs and $f:\left(M_{1}, p_{1}\right) \rightarrow\left(M_{2}, p_{2}\right)$ a map in $\mathcal{Q}$. This induces a map $f^{-1}: \mathcal{T}_{M_{2}} \rightarrow \mathcal{T}_{M_{1}}$ that takes elements of $\mathcal{B}_{p_{2}}$ to $\mathcal{B}_{p_{1}}$. The result follows from Note 6.2.

In what follows, the exact relation between the direct sum of the cohomology ring of certain submanifolds and the $p$-local face rings is clarified.

Theorem 6.10. Let $(M, p)$ be a quasitoric pair. Then for $j \geqslant 0$

$$
L_{j} Q(M, p) \cong L_{j+1} Q(R(p)) \cong \bigoplus_{F \in \widehat{\mathcal{F}}(p)} L_{j+1} Q(R(F)) .
$$

Proof. For each $F \in \widehat{\mathcal{F}}$ let $M_{F}$ be the corresponding quasitoric manifold and we note that such manifolds are basis elements in the topology $\mathcal{T}_{M}$. We have

$$
\begin{aligned}
L_{j+1} Q(R(p)) & =L_{j+1} Q\left(\bigotimes_{F \in \widehat{\mathcal{F}}(p)} R(F)\right) & & \text { by Definition } \\
& \cong \bigoplus_{F \in \widehat{\mathcal{F}}(p)} L_{j+1} Q(R(F)) & & \text { by (iii) of Theorem } 4.4 \\
& \cong \bigoplus_{F \in \widehat{\mathcal{F}}(p)} L_{j+1} Q\left(H^{*}\left(M_{F} ; R\right)\right) & & \text { by Theorem } 4.9 .
\end{aligned}
$$

By Definition 6.1 and the observation above, $\mathfrak{F}(U ; R)$ is the cohomology ring of $M_{F}$ and so $\bigoplus_{F \in \widehat{\mathcal{F}}(p)} L_{j+1} Q\left(H^{*}\left(M_{F} ; R\right)\right)$ is precisely $\bigoplus_{U \in \mathcal{B}_{p}} L_{j+1} Q(\mathfrak{F}(U))$ and the result follows. 
In regards to Definition 6.7 and Theorem 6.10, the case of interest will occur when $j=1$ because the isomorphism of these higher derived functors holds in this range and specific calculations were made for the second higher derived functor of the indecomposable functor [AL3, AL4] cf., Theorems 4.6 and 4.9.

\section{Application}

In this section we consider the polyhedra that result from applying a double vertex truncation as described in $[\mathbf{B E M P P}]$ and $[\mathbf{B P 1}]$. Pictures of each of these three dimensional simple convex polytopes are as follows:

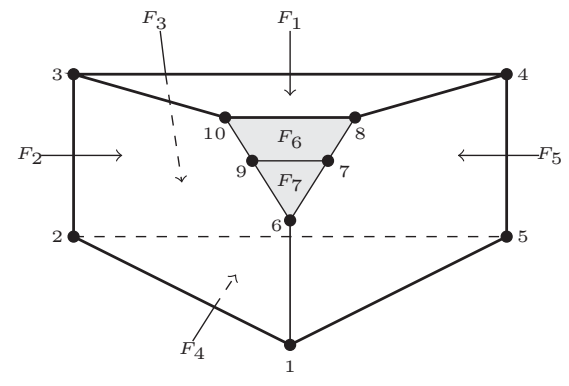

$P_{1}$

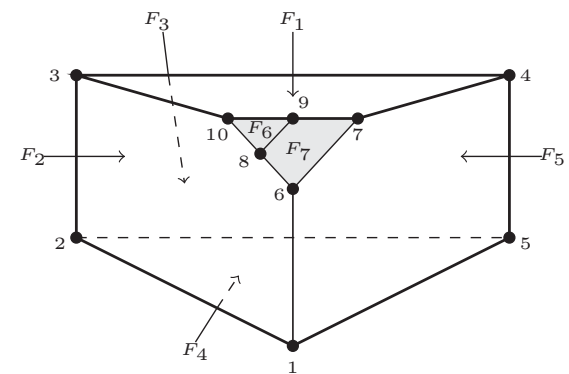

$P_{2}$

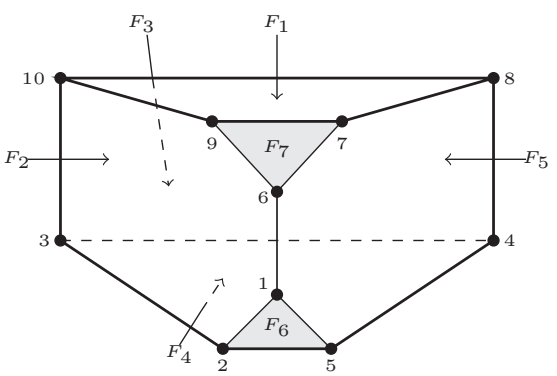

$P_{3}$

For the sake of brevity we write $P_{1}=6^{2} 4^{3} 3^{2}=P_{3}$ and $P_{2}=6^{1} 5^{2} 4^{2} 3^{2}$ taking note that the powers count the number of $m$-gons. For instance, $6^{2}$ means two 6 -gons.

These polytopes serve as the orbit spaces for the quasitoric manifolds $M_{1}, M_{2}$ and $M_{3}$; all of which are diffeomorphic to $\#_{4} \mathbb{C} P^{3}$. We also note that there is no face preserving map from either $P_{1}$ or $P_{3}$ to $P_{2}$ and furthermore, these polytopes are not combinatorially equivalent. A simple application of (iii) of Theorem 4.6 gives the following:

Proposition 7.1. Let $P$ be any one of the $P_{i}$ for $i=1,2,3$ obtained by a double vertex truncation on the prism, then $L_{j} Q(R(P))$ are $R$-free modules and

$$
L_{j} Q(R(P))= \begin{cases}\left.\operatorname{span}_{R}\left\{a_{1}, \ldots, a_{6}\right\}|| a_{k} \mid=4\right\}, & j=1, \\ \left.\operatorname{span}_{R}\left\{b_{1}, \ldots, b_{16}\right\}|| b_{k} \mid=6\right\}, & j=2 .\end{cases}
$$


Observe that these higher derived functors cannot detect, at least by the calculations known to the authors, the difference in these polyhedra when considered individually. The reason for this is that the trivial intersections of the facets are not fine enough, in this context, to see the differences. However, the $p$-local face ring $(\S 6)$ can detect the more subtle differences as seen by the intersections along the vertices. Furthermore, the presheaf theoretic approach allows for us to bypass the difficulty imposed by the lack of a map of quasitoric manifolds that descends to a map of orbit spaces.

Theorem 7.2. The quasitoric pairs $\left(M_{1}, p_{1}\right)$ and $\left(M_{2}, p_{2}\right)$ are not equivalent in $\mathcal{Q}$ for any $p_{1} \in \pi_{1}^{-1}\left(V\left(P_{1}\right)\right)$ and $p_{2} \in \pi_{2}^{-1}\left(V\left(P_{2}\right)\right)$.

Proof. According to the second isomorphism of Theorem 6.10, the rank of $L_{1} Q\left(M_{i}, p_{i}\right)$ is the sum of the ranks of $L_{2} Q(R(F))$ where $F \in \widehat{\mathcal{F}}\left(p_{i}\right)$. Any facet of $P_{1}$ is either a 3,4 or 6-gon and similarly, any facet of $P_{2}$ is either a 3,4,5 or 6-gon. From this and the table of Corollary 4.8 we determine that $r k\left(L_{1} Q\left(M_{1}, p_{1}\right)\right)$ is even for all $p_{1}$. Referring to Tables 1 and 2 in the second appendix we see that $r k\left(L_{1} Q\left(M_{2}, 3\right)\right)=21$.

\section{Appendix A. Formulas for the ranks of the first and second derived functors of $R\left(K_{m}\right)$}

Theorem A.1. Let $K_{m}$ be the simplicial complex dual to an $m$-gon. Then for $m \geqslant 4$ we have

$$
r k\left(L_{1} Q\left(R\left(K_{m}\right)\right)\right)=\frac{m(m-3)}{2} .
$$

Proof. By part (ii) of Theorem 4.6 the rank of the first derived functor is the $R$-span of the relations of the face ring of $K_{m}$. Since the relations are linearly independent, the rank is the number of relations. This calculation is equivalent to finding the number of non-existing edges between pairs of vertices in the $m$-gon. Given $m$ vertices, there are exactly $\left(\begin{array}{c}m \\ 2\end{array}\right)$ ways to form a pair of vertices and there are $m$ edges, so we have:

$$
\begin{aligned}
r k\left(L_{1} Q\left(R\left(K_{m}\right)\right)\right) & =\left(\begin{array}{c}
m \\
2
\end{array}\right)-m \\
& =\frac{m(m-1)}{2}-m \\
& =\frac{m}{2}[m-1-2] \\
& =\frac{m(m-3)}{2} .
\end{aligned}
$$

Before dealing with the second derived functor we introduce a mechanism to assist with counting relations among relations. An angle in the $m$-gon is the union of two non-existent edges with a common vertex. Namely, if $\{a, b\}$ and $\{b, c\}$ are non-edges in the $m$-gon, then the angle would be $\{a, b\} \cup\{b, c\}$. It is not asserted that this union is a simplex; it is not. Angles can be visualized; below is the angle $\{1,3\} \cup\{3,5\}$ in 
the 5-gon:

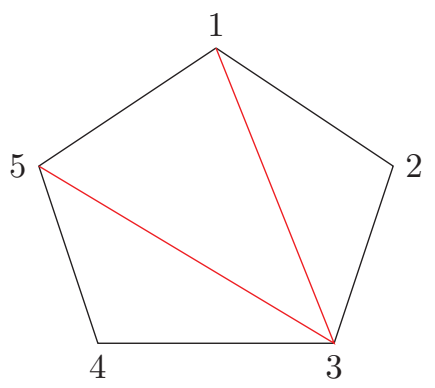

More is true, angles encode relations among relations. For the 5-gon, as above, there is a relation among relations: $\left(x_{1} x_{3}\right) x_{5}-\left(x_{3} x_{5}\right) x_{1}$ and this is equivalent to the angle described above with additional structure. Namely, by completing an angle into a triangle we mean filling in the missing edge so that the vertices that give the angle are now the vertices of the boundary of a two simplex. In the case that an angle is completed in this way, one obtains a triangle. More generally, suppose there are two intersecting relations in the $m$-gon, $r_{1}=x_{i} x_{j}$ and $r_{2}=x_{j} x_{k}$, then the corresponding relation among relations is $\rho=\left(x_{i} x_{j}\right) x_{k}-\left(x_{j} x_{k}\right) x_{i}$. Completing the angle $\{i, j\} \cup\{j, k\}$ gives a corresponding triangle in the $m$-gon which we label as $T(\rho)$.

Some vocabulary will be useful in the sequel. For $k>1$, let $\mathcal{R}(k)$ denote the set of higher order relations for the $m$-gon. For instance, $\mathcal{R}(2)$ is the set of relations among relations, whereas $\mathcal{R}(3)$ would be the set of relations among relations among relations. With this in mind, suppose $\mathcal{R}(2)=\left\{\rho_{1}, \ldots, \rho_{l}\right\}$, then by following the construction above there is a set of triangles $\left\{T\left(\rho_{1}\right), \ldots, T\left(\rho_{l}\right)\right\}$ obtained by completing the angles.

In this context, completing an angle into a triangle is equivalent to analyzing the linear dependence among the elements of $\mathcal{R}(2)$. For example, the following triangle in the 6-gon

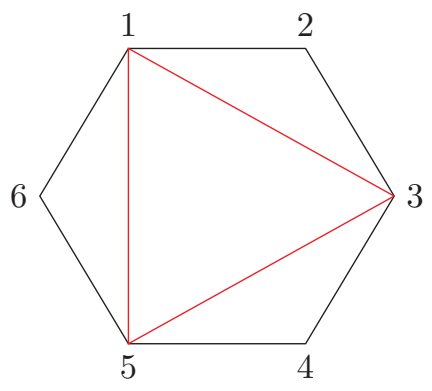

encodes the linear dependence of the $\rho_{i}$ in $\mathcal{R}(2)$ :

$$
\begin{aligned}
& \rho_{1}=\left(x_{1} x_{3}\right) x_{5}-\left(x_{3} x_{5}\right) x_{1}, \\
& \rho_{2}=\left(x_{1} x_{3}\right) x_{5}-\left(x_{1} x_{5}\right) x_{3}, \\
& \rho_{3}=\left(x_{1} x_{5}\right) x_{3}-\left(x_{3} x_{5}\right) x_{1} .
\end{aligned}
$$

A simple verification shows that $\rho_{3}=\rho_{1}-\rho_{2}$. 
We now compute the rank of the second derived functor.

\section{Theorem A.2.}

$$
r k\left(L_{2} Q\left(R\left(K_{m}\right)\right)\right)= \begin{cases}0 & m=3 \\ \frac{m(m-2)(m-4)}{3} & m>3 .\end{cases}
$$

Proof. For $m=3$ there are no relations among relations; hence, the second derive functor is trivial. For $m=4$ there is a relation among relations but it comes from two relations that have trivial intersection; hence the second derived functor is zero by Theorem 4.6. We now focus on the cases $m>4$. By the discussion above it will suffice to first count all the relations among relations which is equivalent to enumerating all the angles in the $m$-gon, then we subtract out all of the linearly dependent ones. This depends on determining and counting the triads. More generally, we must find all sequences $j_{1}, j_{2}, j_{3}$ that satisfy the conditions:
i) $1 \leqslant j_{2} \leqslant m$
ii) $1 \leqslant j_{1}<j_{3} \leqslant m$
iii) $j_{1} \neq j_{2}+1, j_{2}-1, j_{3} \neq j_{2}+1, j_{2}-1$.

Fix $j_{2}$, then we have to choose two elements, $j_{1}$ and $j_{3}$ satisfying conditions (i) and (ii) above, hence there are $m-3$ such vertices. The total number of relations among relations is given by:

$$
m\left(\begin{array}{c}
m-3 \\
2
\end{array}\right)=m\left[\frac{(m-3)(m-4)}{2}\right]=\frac{m(m-3)(m-4)}{2} .
$$

By the discussion above, determining and counting the triads is equivalent to finding all sequences $j_{1}, j_{2}, j_{3}$ where:

i) $1 \leqslant j_{1}<j_{2}<j_{3} \leqslant m$

ii) $j_{1}+1<j_{2}, j_{2}+1<j_{3}$

iii) if $j_{1}=1$ then $j_{3} \neq m$.

For a fixed $j_{1}=j$ with $2 \leqslant j \leqslant m-4$ we can count the number of unions of two non-existent edges using the following table:

\begin{tabular}{|c||c|c|c|c|c|c|}
\hline$j_{2}$ & $j+2$ & $j+3$ & $j+4$ & $\cdots$ & $m-3$ & $m-2$ \\
\hline$j_{3}$ & $j+4$ & & & $\cdots$ & & \\
& $j+5$ & $j+5$ & & $\cdots$ & & \\
& $j+6$ & $j+6$ & $j+6$ & $\cdots$ & & \\
& $\vdots$ & $\vdots$ & $\vdots$ & $\vdots$ & & \\
& $m-1$ & $m-1$ & $m-1$ & $\cdots$ & $m-1$ & \\
& $m$ & $m$ & $m$ & $\cdots$ & $m$ & $m$ \\
\hline \hline totals & $m-j-3$ & $m-j-4$ & $m-j-5$ & $\cdots$ & 2 & 1 \\
\hline
\end{tabular}


Summing the totals and using the formula for the sum of squares we have:

$$
\begin{aligned}
\sum_{i=1}^{m-j-3}(m-j-2-i) & =\sum_{i=1}^{m-j-3}(m-j-2)-\sum_{i=1}^{m-j-3} i \\
& =(m-j-3)(m-j-2)-\frac{1}{2}(m-j-3)(m-j-2) \\
& =\frac{1}{2}(m-j-3)(m-j-2) .
\end{aligned}
$$

We add for $2 \leqslant j \leqslant m-4$

$$
\begin{aligned}
\sum_{j=2}^{m-4} \frac{1}{2}(m-j-3)( & m-j-2)=\sum_{j=1}^{m-5} \frac{1}{2}(m-j-4)(m-j-3) \\
= & \frac{1}{2} \sum_{j=1}^{m-5}\left(m^{2}-7 m+12\right)+\frac{1}{2} \sum_{j=1}^{m-5} j^{2}+\frac{1}{2}(7-2 m) \sum_{j=1}^{m-5} j \\
= & \frac{1}{2}(m-5)\left(m^{2}-7 m+12\right)+\frac{1}{2}\left[\frac{1}{6}(m-5)(m-4)(2 m-9)\right] \\
& +(7-2 m)\left[\frac{1}{4}(m-5)(n-4)\right] \\
= & \frac{1}{2}(m-5)(m-4)(m-3)+\frac{1}{12}(m-5)(m-4)(2 m-9) \\
& +\frac{1}{4}(7-2 m)(m-5)(m-4) \\
= & \frac{1}{12}(m-5)(m-4)[6(m-3)+(2 m-9)+3(7-2 m)] \\
= & \frac{1}{12}(m-5)(m-4)(2 m-6) \\
= & \frac{1}{6}(m-5)(m-4)(m-3) .
\end{aligned}
$$

In the case of $j_{1}=1$ we have the following table:

\begin{tabular}{|c||c|c|c|c|c|c|}
\hline$j_{2}$ & 3 & 4 & 5 & $\cdots$ & $m-4$ & $m-3$ \\
\hline$j_{3}$ & 5 & & & $\cdots$ & & \\
& 6 & 6 & & $\cdots$ & & \\
& 7 & 7 & 7 & $\cdots$ & & \\
& $\vdots$ & $\vdots$ & $\vdots$ & $\vdots$ & & \\
& $m-2$ & $m-2$ & $m-2$ & $\cdots$ & $m-2$ & \\
& $m-1$ & $m-1$ & $m-1$ & $\cdots$ & $m-1$ & $m-1$ \\
\hline totals & $m-5$ & $m-6$ & $m-7$ & $\cdots$ & 2 & 1 \\
\hline
\end{tabular}


Summing the totals we have:

$$
\begin{aligned}
\sum_{i=1}^{m-5}(m-4-i) & =\sum_{i=1}^{m-5}(m-4)-\sum_{i=1}^{m-5} i \\
& =(m-4)(m-5)-\frac{1}{2}(m-5)(m-4) \\
& =\frac{1}{2}(m-5)(m-4) .
\end{aligned}
$$

Adding formulas (A.2) and (A.3) we have

$$
\begin{aligned}
\frac{1}{6}(m-5)(m-4)(m-3)+\frac{1}{2}(m-5)(m-4) & =\frac{1}{6}(m-5)(m-4)[(m-3)+3] \\
& =\frac{1}{6} m(m-4)(m-5) .
\end{aligned}
$$

Thus the number of generators of $L_{2} Q\left(R\left(K_{m}\right)\right)$ is given by (A.1)-(A.4) and we obtain:

$$
\begin{aligned}
r k\left(L_{2} Q\left(R\left(K_{m}\right)\right)\right) & =\frac{1}{2} m(m-3)(m-4)-\frac{1}{6} m(m-4)(m-5) \\
& =\frac{1}{6} m(m-4)[3(m-3)-(m-5)] \\
& =\frac{1}{3} m(m-2)(m-4) .
\end{aligned}
$$

\section{Appendix B. Ranks of the $p$-local face rings of $M_{1}$ and $M_{2}$}

For clarification we list the faces of each polytope that could contribute to the rank of the second derived functor. We recall that 0 -faces, 1-faces and 2-faces do not contribute any generators to the rank of the second derived functor (see Theorem A.2).

Table 1: Classification of the facets of $P_{1}$ and $P_{2}$.

\begin{tabular}{|c|c|c|}
\hline & $P_{1}$ & $P_{2}$ \\
\hline 3-gon & $F_{4}, F_{7}$ & $F_{4}, F_{6}$ \\
\hline 4-gon & $F_{1}, F_{3}, F_{6}$ & $F_{3}, F_{7}$ \\
\hline 5-gon & & $F_{1}, F_{5}$ \\
\hline 6-gon & $F_{2}, F_{5}$ & $F_{2}$ \\
\hline
\end{tabular}

Table 2 lists all the vertices and the facets that contain them for $P_{1}$ and $P_{2}$ pictured in $\S 7$. Columns 3 and 5 are the ranks of the second derived functors for each facet that contains each vertex listed in column 1 . Rows $3,5,7,9,11,13,15,17,19$ and 21 are the ranks of the $p$-local face rings of $M_{1}$ and $M_{2}$ at the given vertex (see Theorem 6.10). 
Table 2: Ranks of the $p$-local face rings for vertices of $M_{1}$ and $M_{2}$.

\begin{tabular}{|c|c|c|c|c|}
\hline \multicolumn{3}{|c|}{$P_{1}$} & \multicolumn{2}{|c|}{$P_{2}$} \\
\hline$p$ & $\widehat{\mathcal{F}}(p)$ & $r k\left(L_{2} Q(R(F))\right)$ & $\widehat{\mathcal{F}}(p)$ & $r k\left(L_{2} Q(R(F))\right)$ \\
\hline \multirow{3}{*}{1} & $F_{2}$ & 16 & $F_{2}$ & 16 \\
\hline & $F_{4}$ & 0 & $F_{4}$ & 0 \\
\hline & $F_{5}$ & 16 & $F_{5}$ & 5 \\
\hline \multicolumn{2}{|c|}{$r k\left(L_{1} Q\left(M_{1}, 1\right)\right)$} & 32 & $r k\left(L_{1} Q\left(M_{1}, 1\right)\right)$ & 21 \\
\hline \multirow{3}{*}{2} & $F_{2}$ & 16 & $F_{2}$ & 16 \\
\hline & $F_{3}$ & 0 & $F_{3}$ & 5 \\
\hline & $F_{4}$ & 0 & $F_{4}$ & 0 \\
\hline \multicolumn{2}{|c|}{$\operatorname{rk}\left(L_{1} Q\left(M_{1}, 2\right)\right)$} & 16 & $r k\left(L_{1} Q\left(M_{1}, 2\right)\right)$ & 21 \\
\hline \multirow{3}{*}{3} & $F_{1}$ & 0 & $F_{1}$ & 5 \\
\hline & $F_{2}$ & 16 & $F_{2}$ & 16 \\
\hline & $F_{3}$ & 0 & $F_{3}$ & 0 \\
\hline \multicolumn{2}{|c|}{$r k\left(L_{1} Q\left(M_{1}, 3\right)\right)$} & 16 & $r k\left(L_{1} Q\left(M_{1}, 21\right)\right)$ & 21 \\
\hline \multirow{3}{*}{4} & $F_{1}$ & 0 & $F_{1}$ & 5 \\
\hline & $F_{3}$ & 0 & $F_{3}$ & 0 \\
\hline & $F_{5}$ & 16 & $F_{5}$ & 5 \\
\hline \multicolumn{2}{|c|}{$r k\left(L_{1} Q\left(M_{1}, 4\right)\right)$} & 16 & $r k\left(L_{1} Q\left(M_{1}, 4\right)\right)$ & 10 \\
\hline \multirow{3}{*}{5} & $F_{3}$ & 0 & $F_{3}$ & 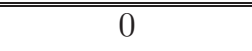 \\
\hline & $F_{4}$ & 0 & $F_{4}$ & 0 \\
\hline & $F_{5}$ & 16 & $F_{5}$ & 5 \\
\hline \multicolumn{2}{|c|}{$\operatorname{rk}\left(L_{1} Q\left(M_{1}, 5\right)\right)$} & 16 & $r k\left(L_{1} Q\left(M_{1}, 5\right)\right)$ & 5 \\
\hline \multirow{3}{*}{6} & $F_{2}$ & 16 & $F_{2}$ & 16 \\
\hline & $F_{5}$ & 16 & $F_{5}$ & 5 \\
\hline & $F_{7}$ & 0 & $F_{7}$ & 0 \\
\hline \multicolumn{2}{|c|}{$r k\left(L_{1} Q\left(M_{1}, 6\right)\right)$} & 32 & $r k\left(L_{1} Q\left(M_{1}, 6\right)\right)$ & 21 \\
\hline \multirow{3}{*}{7} & $F_{5}$ & $\overline{16}$ & $\begin{array}{l}F_{1} \\
\end{array}$ & 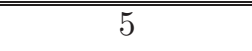 \\
\hline & $F_{6}$ & 0 & $F_{5}$ & 5 \\
\hline & $F_{7}$ & 0 & $F_{7}$ & 0 \\
\hline \multicolumn{2}{|c|}{$r k\left(L_{1} Q\left(M_{1}, 7\right)\right)$} & 16 & $r k\left(L_{1} Q\left(M_{1}, 7\right)\right)$ & 10 \\
\hline \multirow{3}{*}{8} & $F_{1}$ & 0 & $\begin{array}{l}F_{2} \\
\end{array}$ & $\overline{16}$ \\
\hline & $F_{5}$ & 16 & $F_{6}$ & 0 \\
\hline & $F_{6}$ & 0 & $F_{7}$ & 0 \\
\hline \multicolumn{2}{|c|}{$r k\left(L_{1} Q\left(M_{1}, 8\right)\right)$} & 16 & $r k\left(L_{1} Q\left(M_{1}, 8\right)\right)$ & 16 \\
\hline \multirow{3}{*}{9} & $F_{2}$ & $\overline{16}$ & (" $F_{1}$ & 5 \\
\hline & $F_{6}$ & 0 & $F_{6}$ & 0 \\
\hline & $F_{7}$ & 0 & $F_{7}$ & 0 \\
\hline \multicolumn{2}{|c|}{$r k\left(L_{1} Q\left(M_{1}, 9\right)\right)$} & 16 & $r k\left(L_{1} Q\left(M_{1}, 9\right)\right)$ & 5 \\
\hline \multirow{3}{*}{10} & $F_{1}$ & 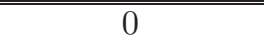 & (" $F_{1}$ & 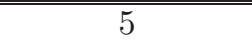 \\
\hline & $F_{2}$ & 16 & $F_{2}$ & 16 \\
\hline & $F_{6}$ & 0 & $F_{6}$ & 0 \\
\hline \multicolumn{2}{|c|}{$r k\left(L_{1} Q\left(M_{1}, 10\right)\right)$} & 16 & $r k\left(L_{1} Q\left(M_{1}, 10\right)\right)$ & 21 \\
\hline
\end{tabular}




\section{References}

[A] Allen D., On the homotopy groups of toric spaces, Homology Homotopy Appl., vol. 10(1), 2008, pp. 437-479.

[AL] Allen D., La Luz J., The higher derived functors of the primitive element functor of quasitoric manifolds, Topology Appl., vol. 158(16), 2011, pp. $2103-2110$.

[AL2] Allen D., La Luz J., Methods in unstable homotopy theory, Surv. Math. Math. Sci., vol. 1(1), 2012 , pp. 1-34.

[AL3] Allen D., La Luz J., On the non-existence of torus actions, Topology Appl., vol. 209, 2016, pp. 347-366.

[AL4] Allen D., La Luz J., Certain generalized higher derived functors associated to quasitoric manifolds, J. Homotopy Relat. Struct., 2017, https://doi.org/10.1007/s40062-017-0186-y.

[BEMPP] Buchstaber V.M., Erokhovets N., Masuda M., Panov T.E., Park S., Cohomological rigidity of manifolds defined by right-angled 3-dimensional polytopes, Arxiv: 1610.07575 v2, 2017.

[BP1] Buchstaber V.M., Panov T.E., Torus Actions and Their Applications in Topology and Combinatorics, University Lecture Series, AMS, 2002.

[BR] Buchstaber V.M., Ray N., Tangential structures on toric manifolds, and connected sums of polytopes, Int. Math. Res. Not. IMRN, 2001.

[BR2] Buchstaber V.M., Ray N., An invitation to toric topology: vertex four of a remarkable tetrahedron. In: Toric Topology (M. Harada et al., eds.) Contemp. Math., vol. 460, Amer. Math Soc., Providence, RI, 1970, pp. $1-27$.

[DJ] Davis M., Januszkiewicz T., Convex polytopes, Coxeter orbifolds and torus actions, Duke Math. J., vol. 62(2), 1991, pp. 417-451.

[OR] Orlik P., Raymond F., Actions of the torus on 4-manifolds I, Trans. Amer. Math. Soc., vol. 152, 1970, pp. 531-559.

David Allen dtallen@bmcc.cuny.edu

Department of Mathematics BMCC CUNY, New York 10007, USA

José La Luz jose.laluz1@upr.edu

Departmento de Matemáticas, Universidad de Puerto Rico, Industrial Minillas 170 Car 174, Bayamón, PR 00959-1919, USA 